\title{
Magnetometers for tracking metallic targets
}

\author{
Niklas Wahlström, Jonas Callmer and Fredrik Gustafsson \\ Division of Automatic Control \\ Linköping University \\ Linköping, Sweden \\ niklas.wahlstrom@gmail.com, callmer@isy.liu.se, fredrik@isy.liu.se
}

\begin{abstract}
Starting from Maxwell's equations, we derive a sensor model for three-axis magnetometers suitable for localization and tracking applications. The model depends on the relative position between the sensor and the target, and a physical magnetic multipole model of the target. Both point targets (far-field) and extended target (near-field) models are provided. The models are validated on data taken from various road vehicles. The suitability of magnetometers for tracking is analyzed in terms of local observability and Cramér Rao lower bound as a function of the sensor positions in a two sensor scenario. Results from field test data indicate excellent tracking of position and velocity of the target, as well as identification of the magnetic target model suitable for target classification.
\end{abstract}

Keywords: Tracking, magnetic dipole, observability.

\section{Introduction}

Tracking and classification of targets are primary concerns in automated surveillance systems. The tracking and classification information can be used for statistical purposes, i.e. counting number of target of a specific type and registration of their velocities and directions of arrival. If a permeable body is placed in a region with a homogeneous magnetic field, it becomes magnetized, which gives rise to an additive induced magnetic field [1]. In this work, the magnetic field of the earth induces a magnetization in a vehicle. If the vehicle is moving, this induced magnetic field can be measured at different positions relative to the vehicle with a stationary magnetometer. This signal depends on the position, velocity and the magnetic signature of the target. This work presents nonlinear statistical signal processing methods to be used with this signal in order to track vehicles.

The complete description of the induced magnetic field caused by a moving magnetized target is given if the position and the magnetic properties of each atom in the vehicle is known. However, this (almost) infinite state space can also be parameterized using the so called multipole expansion [1, 2], which is an orthogonal expansion of the magnetic field. Its coefficients are known as the magnetic moments. The highly nonlinear basis functions of this expansion decay more and more rapidly with the distance from the target. Generally, this expansion can be truncated and far away enough (approximately the characteristic length of the target) only the lowest order term of the expansion is of importance. This term is known as the magnetic dipole field and its coefficient as the magnetic dipole moment.

This dipole model has been used by Birsan [3, 4] for target tracking, where simulations of known position and magnetic dipole moment have been used to estimate these parameters. Tracking and estimation were performed using an unscented Kalman filter [3] and an unscented particle filter [4]. Furthermore, several studies have been done exploring the use of underwater magnetometers for tracking of vessels [5, 6, 7], where [7] has used the dipole model not only for localizing the vessel, but also for estimating the positions of the sensors. In [6] a Bayesian match-field approach has been used, which takes the attenuation of the seawater into consideration.

In [4], the problem with determination of arrival direction due to the nonlinearity of the sensor model has been addressed, which has been solved with a Monte Carlo based approach known as the particle filter. However, in this work, the vehicle is assumed to follow a straight road and only two directions of arrival have to been considered, which can be solved by initializing two Extended Kalman Filters. Furthermore, the works using the dipole model $[3,4,7]$ are based on simulations, whereas this work will compare the theory with experimental data. In addition, in [4] the problem with lack of observability for an one-sensor scenario has been addressed. Our work will more precisely describe this unobservable manifold. Finally, the optimal sensor deployment is analyzed in terms of local observability and Cramér Rao lower bound as a function of the sensor positions in a two-sensor scenario. 
The paper outline is as follows: Section 2 describes the sensor model and compares it with other common sensors. A validation of this sensor model based on experimental data is provided in Section 3. In Section 4 observability and sensor deployment is discussed and in Section 5 results from a target tracking experiment is provided. The paper concludes with conclusions and appendix.

\section{Theoretical Sensor Model}

A permeable object in a homogeneous magnetic field gives rise to an induced magnetic field. In order to design a good statistical signal processing method for tracking and classifying this target, physical insight into how this field depends on the properties of the target as well as the distance and direction from it has to be achieved. If the distance from the object is large in comparison with its characteristic length, the induced magnetic field $\mathbf{B}(\mathbf{r})$ at position $\mathbf{r}$ relative to the object can be described as a dipole field. An expression of this field can be derived from Maxwell's equations (see Appendix 6) and in Cartesian coordinates it is

$$
\mathbf{B}(\mathbf{r})=\frac{\mu_{0}}{4 \pi} \frac{3(\mathbf{r} \cdot \mathbf{m}) \mathbf{r}-r^{2} \mathbf{m}}{r^{5}},
$$

where $\mathbf{m}$ is the magnetic dipole moment of the object and $r=|\mathbf{r}|$. In the derivation of this term, the Taylor expansion of the potential $1 / r$ has been truncated (see Equation (38)). By going further with this expansion, higher moments of the magnetic vector potential are found. This expansion is known as the multipole expansion. The $l$ th magnetic moment $m_{i_{1} \ldots i_{l}}$ is a symmetric traceless tensor of $l$ th order having $2 l+1$ independent components. The magnetic field of the $l$ th multipole may be expressed as (for derivation see [8])

$$
\mathbf{B}^{(l)}(\mathbf{r})=\frac{\mu_{0}}{4 \pi} \frac{(2 l+1)\left(\mathbf{r} \cdot \mathbf{M}^{(l)}\right) \mathbf{r}-l r^{2} \mathbf{M}^{(l)}}{r^{l+4}},
$$

where the following vector has been introduced

$$
M_{i}^{(l)}=\sum_{i_{1}=1}^{3} \cdots \sum_{i_{l-1}=1}^{3} \frac{x_{i_{1}} \cdots x_{i_{l-1}}}{r^{l-1}} \frac{1}{l !} m_{i i_{1} \ldots i_{l-l}} .
$$

By setting $l=1$ one can easily compute the magnetic dipole in (1). The total magnetic field is then given by

$$
\mathbf{B}(\mathbf{r})=\sum_{l=1}^{\infty} \mathbf{B}^{(l)}(\mathbf{r}) .
$$

The $r$-dependences of the field for the different multipoles are $r^{-3}, r^{-4}, r^{-5}$ etc. and at sufficiently large distances the field is dominated by the dipole term. Generally the series (4) can be truncated to arbitrary many multipoles. With a truncation of the $p$ th first multipoles, one gets

$$
\mathbf{B}(\mathbf{r})=\sum_{l=1}^{p} \mathbf{B}^{(l)}(\mathbf{r})+\mathcal{O}\left(\frac{1}{r^{p+3}}\right),
$$

since the lowest term not included in the expansion will dominate the truncation error at large distances. Thus, the multipole expansion also gives a hint how good this truncation is which can be used for variance and bias compensation of the measurement.

In the signal processing framework, a sensor model of a time-invariant system is given on the standard form

$$
\mathbf{y}_{k}=\mathbf{h}\left(\mathbf{x}_{k}\right)+\mathbf{e}_{k},
$$

where $\mathbf{y}_{k}$ is the measurement, $\mathbf{x}_{k}$ is the state of the system and $\mathbf{e}_{k}$ is measurement noise, all at time instant $k T_{s}, T_{s}$ being the sample period. Let $\mathbf{r}_{k}$ be the position of the target relative to the sensor and $\mathbf{m}_{k}$ its magnetic dipole moment. By choosing $p=1$ in (5), one gets the sensor model

$\mathbf{y}_{k}=\mathbf{h}\left(\mathbf{r}_{k}, \mathbf{m}_{k}\right)+\mathbf{e}_{k}=\frac{\mu_{0}}{4 \pi} \frac{3\left(\mathbf{r}_{k} \cdot \mathbf{m}_{k}\right) \mathbf{r}_{k}-\left|\mathbf{r}_{k}\right|^{2} \mathbf{m}_{k}}{\left|\mathbf{r}_{k}\right|^{5}}+\mathbf{e}_{k}$

where $\mathbf{r}_{k}$ and $\mathbf{m}_{k}$ are the states of the system. This will be a good far-field model since the model error to signal ratio decays as $\mathcal{O}\left(r^{-4}\right)^{2} / \mathcal{O}\left(r^{-3}\right)^{2}=\mathcal{O}\left(r^{-2}\right)$. Furthermore, it is instructive to express (1) in spherical coordinates $(r, \theta, \phi)$ with $\mathbf{m}$ being the zenith direction

$$
\begin{aligned}
& B_{r}(\mathbf{r})=\frac{\mu_{0}}{4 \pi} \frac{2 m \cos (\theta)}{r^{3}} \\
& B_{\theta}(\mathbf{r})=\frac{\mu_{0}}{4 \pi} \frac{m \sin (\theta)}{r^{3}} \\
& B_{\phi}(\mathbf{r})=0,
\end{aligned}
$$

where $m=|\mathbf{m}|$. Note that these equations reveal the real information in the three-axis magnetometer. Only a two-dimensional subspace affects the sensor, which is parameterized by the range $r$ and the bearing $\theta$ to the target (relative its own magnetic dipole moment). One can compare this sensor model with other common sensors in tracking and localization applications that also provide range and bearing information:

- A radar measures range and bearing explicitly, while the magnetometer model provides this information implicitly. It should therefore contain similar information. The radar signal decays as $\mathcal{O}\left(r^{-4}\right)$, and the usual thresholding gives a binary SNR (either the target is detected or it is not) which depends on range. The SNR for the magnetometer decays continuously as $\mathcal{O}\left(r^{-6}\right)$ and in contrast to a radar, the emitted power of the earth magnetic field cannot be increased to improve SNR.

- Time of arrival (TOA) measures the time of flight between two points, which is linear in range. The signal decays approximately as $\mathcal{O}\left(r^{-2}\right)$, and similar to the radar the SNR is quantized in the signal strength. 
- Received Signal Strength (RSS) measures the signal level (in dB usually), and this level decays logarithmically in range, so SNR is approximately $\mathcal{O}(-\log (r))$.

\section{Sensor Model Validation}

In order to validate the far-field sensor model (7) real experimental data has been collected with a Xsens magnetometer [9]. In this section, the measurement noise of this data will be analyzed. Furthermore, the experimental setup will be described as well as the methodology of the validation and the results.

\subsection{Noise Distribution}

Without any targets, the magnetometer measures only the present homogeneous magnetic field (the magnetic field of the earth) together with noise. The homogeneous magnetic field can be regarded as a constant bias, and thus be subtracted from the original signal. According to [10] the noise of the magnetometer [9] is white Gaussian, i.e. its samples are i.i.d. and normal distributed. Such a stochastic process is uniquely defined by its covariance matrix (since a bias of the sensor already has been subtracted). This symmetric $3 \times 3-$ matrix is estimated to be

$$
R=10^{-20}\left(\begin{array}{ccc}
0.2150 & 0.0010 & 0.0252 \\
0.0010 & 0.0549 & 0.0011 \\
0.0252 & 0.0011 & 0.0954
\end{array}\right)
$$

where the unit of the measurement is Tesla.

\subsection{Experimental Setup and Velocity model}

The magnetometer has been placed close to a straight road measuring the induced magnetic field due to vehicles passing by. The sensor setup is presented in Figure 1 .

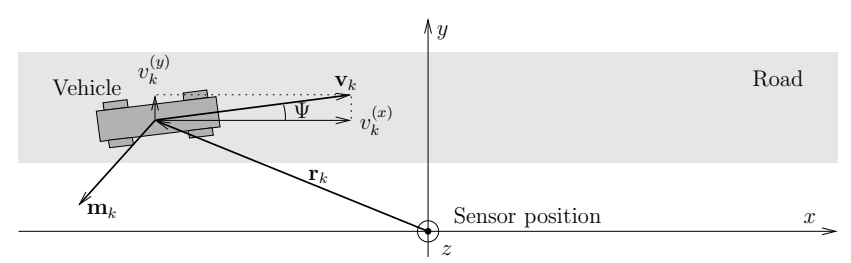

Figure 1: Sensor setup for model validation. $\mathbf{r}_{k}$ is a vector from the sensor to the vehicle, $\mathbf{v}_{k}$ is the velocity of the vehicle and $\mathbf{m}_{k}$ is the magnetic moment of the vehicle. All vectors are three dimensional and given in the global Cartesian coordinate system $x-y-z$.

Since the road is straight, a constant velocity motion model is assumed for the vehicle. This gives the following 9-dimensional discrete state space model:

$$
\begin{aligned}
\mathbf{r}_{k+1} & =\mathbf{r}_{k}+T \mathbf{v}_{k}+\frac{T^{2}}{2} \mathbf{w}_{k} \\
\mathbf{v}_{k+1} & =\mathbf{v}_{k}+T \mathbf{w}_{k} \\
\mathbf{m}_{k+1} & =\mathbf{m}_{k} \\
\mathbf{y}_{k} & =\frac{\mu_{0}}{4 \pi} \frac{3\left(\mathbf{r}_{k} \cdot \mathbf{m}_{k}\right) \mathbf{r}_{k}-\left|\mathbf{r}_{k}\right|^{2} \mathbf{m}_{k}}{\left|\mathbf{r}_{k}\right|^{5}}+\mathbf{e}_{k},
\end{aligned}
$$

where $\mathbf{w}_{k} \sim \mathcal{N}(\mathbf{0}, \mathbf{Q})$ is white Gaussian process noise and $\mathbf{e}_{k} \sim \mathcal{N}(\mathbf{0}, \mathbf{R})$ is white Gaussian measurement noise, given by $(9)$.

\subsection{NLS for getting a Ground Truth So- lution}

Since all vehicles in our experiment pass the sensor with constant velocity, this prior knowledge enables a dedicated procedure to generate the ground truth with high accuracy. That is, assume $\mathbf{v}_{k+1}=\mathbf{v}_{k}$ and thus $\mathbf{w}_{k}=\mathbf{0}$ in (10). Set

$$
\tilde{\mathbf{h}}_{k}(\mathbf{x})=\mathbf{h}\left(\mathbf{r}_{0}+k T_{s} \mathbf{v}_{0}, \mathbf{m}\right)
$$

The NLS methodology then gives

$$
\begin{aligned}
\hat{\mathbf{x}} & =\arg \min _{\mathbf{x}} V(\mathbf{x}) \\
V(\mathbf{x}) & =\sum_{k=1}^{N}\left(\mathbf{y}_{k}-\tilde{\mathbf{h}}_{k}(\mathbf{x})\right)^{T} R^{-1}\left(\mathbf{y}_{k}-\tilde{\mathbf{h}}_{k}(\mathbf{x})\right)
\end{aligned}
$$

In this framework, also the covariance of the optimum $\hat{\mathbf{x}}$ can be estimated. Since $\boldsymbol{\nabla} V=\mathbf{0}$ at optimum, the covariance can be approximated as

$$
\operatorname{Cov}(\hat{\mathbf{x}}) \approx\left(\sum_{k=1}^{N} \nabla \tilde{\mathbf{h}}_{k}(\hat{\mathbf{x}})^{T} R^{-1} \nabla \tilde{\mathbf{h}}_{k}(\hat{\mathbf{x}})\right)^{-1} .
$$

Furthermore, according to the result in Section 4.1 this system is not observable, which could be solved with a second sensor. However, in this sensor model validation an assumption has had to be made and the velocity is assumed to be equal to $10 \mathrm{~m} / \mathrm{s}$ for all vehicles. In order to implement this assumption, the velocity $\mathbf{v}_{0}$ has been parameterized with its magnitude, yaw and pitch angle $\left(v_{0}, \Psi, \theta\right)$ and the assumption $v_{0}=10$. The NLS problem can now be solved with the Gauss-Newton algorithm and the results are summarized in Table 1, 2 and 3 for the vehicles presented in Figure 3.

From Table 1 it can be concluded that the signal from Vehicle 6 produces the best fit with the model and Vehicle 1 the worst. This can be explained by looking at the pictures in Figure 3. Here, it can be stated that Vehicle 1 is much closer to the sensor in comparison with the characteristic length of the object than Vehicle 6 . In Figure 2 a plot illustrates the excellent results for Vehicle 6. Furthermore, from Table 2, the values of $\hat{\Psi}$ and 
Table 1: The cost function, and the estimated yaw $\hat{\Psi}$ and pitch $\hat{\theta}$ angle with standard deviation in parenthesis.

\begin{tabular}{|l|l|l|l|l|}
\hline $\mathrm{Nr}$ & $V(\hat{\mathbf{x}})$ & $N$ & Yaw $\hat{\Psi}\left[^{\circ}\right]$ & Pitch $\hat{\theta}\left[^{\circ}\right]$ \\
\hline 1 & $7.4 \mathrm{e}+04$ & 31 & $-11.8(0.2)$ & $-20.6(0.3)$ \\
2 & $2.5 \mathrm{e}+04$ & 97 & $179(0.2)$ & $2.6(0.1)$ \\
\hline 3 & $2.5 \mathrm{e}+03$ & 43 & $169(1.5)$ & $14.0(1.0)$ \\
4 & $5.5 \mathrm{e}+02$ & 29 & $6.4(0.8)$ & $3.4(0.5)$ \\
5 & $3.3 \mathrm{e}+02$ & 27 & $3.5(1.5)$ & $2.0(0.6)$ \\
6 & $1.0 \mathrm{e}+02$ & 23 & $176(2.7)$ & $-0.3(1.6)$ \\
\hline
\end{tabular}

Table 2: The estimated the initial position $\hat{\mathbf{r}}_{0}$ in Cartesian coordinates with standard deviation in parenthesis.

\begin{tabular}{|l|llllll|}
\hline $\mathrm{Nr}$ & \multicolumn{5}{|c|}{ Estimated initial position $\hat{\mathbf{r}}_{0}[\mathrm{~m}]$} \\
\hline 1 & -6.7, & 5.7, & -7.3 & $(0.05$, & 0.03, & $0.04)$ \\
2 & 34, & 15, & -7.6 & $(0.05$, & 0.11, & $0.10)$ \\
\hline 3 & 17, & 6.3, & 1.3 & $(0.15$, & 0.40, & $0.39)$ \\
4 & -12, & 3.5, & -0.8 & $(0.04$, & 0.16, & $0.09)$ \\
5 & -11, & 3.7, & 0.2 & $(0.08$, & 0.28, & $0.09)$ \\
6 & 7.4, & 5.1, & -1.0 & $(0.23$, & 0.34, & $0.3)$ \\
\hline
\end{tabular}

Table 3: The estimated magnetic dipole moment $\hat{\mathbf{m}}$ in Cartesian coordinates with standard deviation in parenthesis.

\begin{tabular}{|l|llllll|}
\hline $\mathrm{Nr}$ & \multicolumn{6}{|c|}{ Estimated magnetic dipole moment $\hat{\mathbf{m}}[\mathrm{J} / \mathrm{T}]$} \\
\hline 1 & 9, & -1.3, & -6 & $(0.05$, & 0.08, & $0.06)$ \\
2 & 260, & -27, & 140 & $(0.91$, & 1.30, & $0.63)$ \\
\hline 3 & 7.6, & 3.1, & -44 & $(1.60$, & 0.40, & $0.38)$ \\
4 & -3, & -0.2, & -3.1 & $(0.04$, & 0.09, & $0.04)$ \\
5 & -1.4, & 0.3, & -1.6 & $(0.03$, & 0.07, & $0.03)$ \\
6 & -1.2, & 0.4, & -1.4 & $(0.09$, & 0.12, & $0.05)$ \\
\hline
\end{tabular}

$\hat{\theta}$ reveal that all the velocities of the vehicles are almost parallel to the $x$-axis, in accordance with the experimental setup (see Figure 1). It can also be stated that Vehicle 1,4 and 5 move in positive $x$-direction, whereas, Vehicle 2, 3 and 6 move in negative $x$-direction, which can be verified in Figure 3 .

From Tables 2 and 3 only the directions of the presented vector quantities are interesting, since the norms will be scaled if another assumption for the velocity is made.

\section{Magnetometer Potential for Localization and Tracking}

In this section limitations and possibilities of the magnetometer will be discussed in terms of observability and Cramér Rao lower bound as a function of the

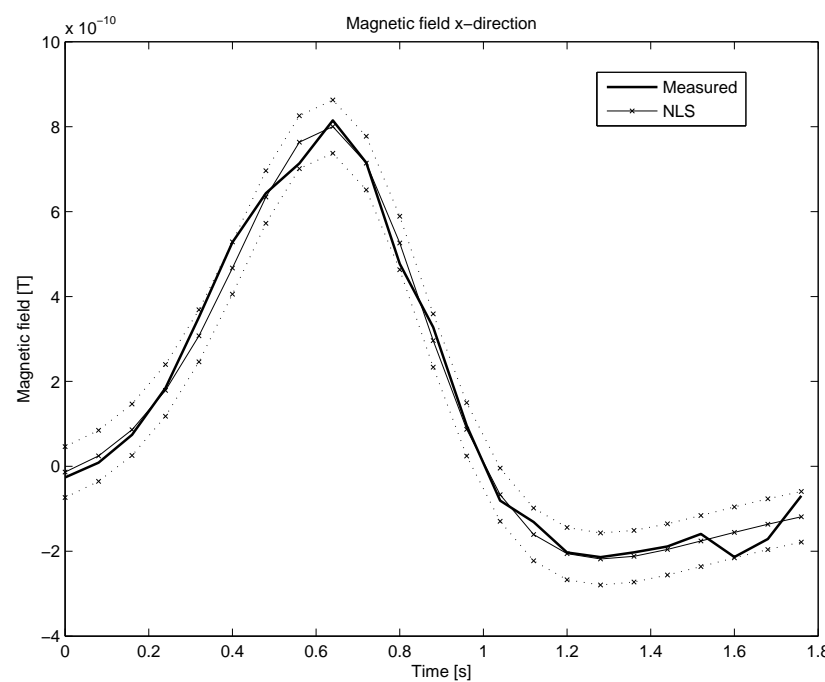

Figure 2: The measured magnetic field $y_{k}^{(x)}$ in the $\mathrm{x}$-direction together with the NLS-estimated value $\tilde{h}_{k}^{(x)}(\hat{\mathbf{x}})$ for Vehicle 6 with a $90 \%$ confidence interval. For the non-linear transformation $\tilde{h}_{k}^{(x)}(\hat{\mathbf{x}})$ a first order Taylor approximation has been used.

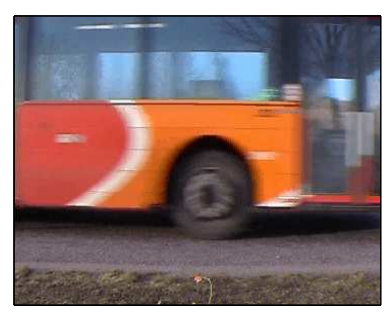

(a) Vehicle 1

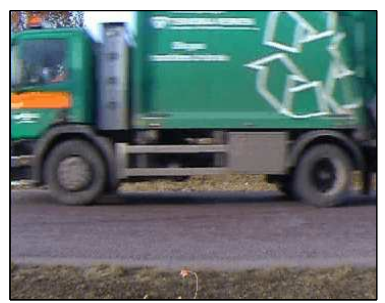

(c) Vehicle 3

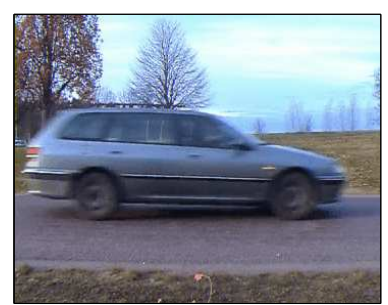

(e) Vehicle 5

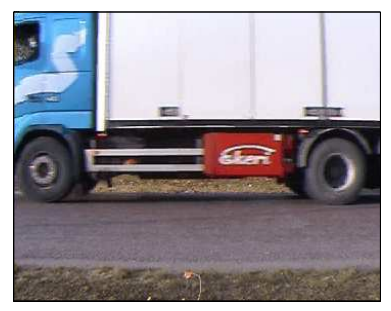

(b) Vehicle 2

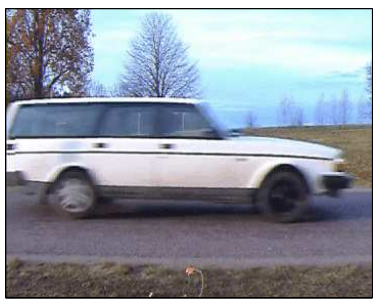

(d) Vehicle 4

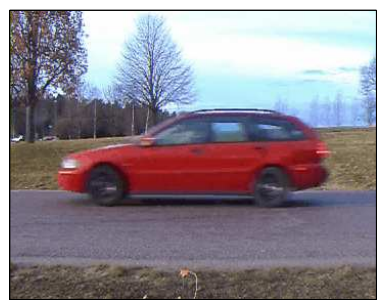

(f) Vehicle 6
Figure 3: The vehicles used in the sensor model validation. 
placement of a second sensor.

\subsection{Single Sensor Observability}

To analyze observability, a local analysis can be performed. Consider the covariance of the NLS estimate (13). This is computed by inverting the information matrix

$$
\mathcal{I}\left(\mathbf{x}^{0}\right)=\sum_{k=1}^{N} \nabla \tilde{\mathbf{h}}_{k}^{T}\left(\mathbf{x}^{0}\right) R^{-1} \nabla \tilde{\mathbf{h}}_{k}\left(\mathbf{x}^{0}\right),
$$

where $\mathbf{x}^{0}$ are the true parameters. Any zero eigenvalues of this matrix makes it singular, which indicates a lack of local observability. The unobservable subspace is spanned by the eigenvectors corresponding to the zero eigenvalues, also known as the kernel of the matrix. This kernel can be computed for a specific setup.

Consider the parameters in Table 4. This will gener-

Table 4: Parameters for a local observability analysis

\begin{tabular}{|ll|lll|}
\hline Parameter & & \multicolumn{3}{|c|}{ Value } \\
\hline Initial position & $\mathbf{r}_{0}[\mathrm{~m}]$ & -3 & 1 & 0 \\
Velocity & $\mathbf{v}_{0}\left[\mathrm{~m} / \mathrm{s}^{2}\right]$ & 1 & 0 & 0 \\
Magn. dipole moment & $\mathbf{m}$ & 1 & 1 & 1 \\
Measurement noise & $R$ & $I_{3}$ & & \\
Samples & $N$ & 60 & & \\
Sample time & $T_{s}$ & 0.1 & \\
\hline
\end{tabular}

ate a scenario where the target is starting at $[-3,1,0]$, following the $x$-axis and ending at $[3,1,0]$.

By parameterizing $\mathbf{x}^{0}=\left[\mathbf{r}_{0}, \mathbf{v}_{0}, \mathbf{m}\right]^{T}$ and computing the eigenvalues of the information matrix (14), this will result in one eigenvalue having significantly less magnitude than the other eigenvalues (see Figure 4 ). The corresponding eigenvector is $[-3,1,0,1,0,0,3,3,3]^{T}$, which equals $\left[\mathbf{r}_{0}, \mathbf{v}_{0}, 3 \mathbf{m}\right]^{T}$. This property is not unique for the chosen $\mathbf{x}^{0}$. In fact, it can be shown that $\mathcal{I}\left(\mathbf{x}^{0}\right) \cdot\left[\mathbf{r}_{0}, \mathbf{v}_{0}, 3 \mathbf{m}\right]^{T}=\mathbf{0}$ for all $\mathbf{x}^{0} \in \mathbb{R}^{9}$. Thus, the kernel is generally given by the following one-dimensional subspace

$$
\operatorname{Ker}\left(\mathcal{I}\left(\mathbf{x}^{0}\right)\right)=\left(\lambda\left[\mathbf{r}_{0}, \quad \mathbf{v}_{0}, 3 \mathbf{m}\right]^{T} \mid \lambda \in \mathbb{R}\right) .
$$

However, due to the nonlinearity, this expression of the unobservable subspace is only valid at the point $\mathbf{x}^{0}$ and can therefore only be regarded as the tangent of the unobservable one-dimensional manifold at this point. Denote this manifold $\mathbf{X}(u)=\left[\mathbf{R}_{0}(u), \mathbf{V}_{0}(u), \mathbf{M}(u)\right]^{T}$, where $u$ is a scalar parameter. For each $u$ there will be a $\lambda(u)$ such that

$$
\frac{d}{d u} \mathbf{X}(u)=\frac{d}{d u}\left(\begin{array}{c}
\mathbf{R}_{0}(u) \\
\mathbf{V}_{0}(u) \\
\mathbf{M}(u)
\end{array}\right)=\lambda(u)\left(\begin{array}{c}
\mathbf{R}_{0}(u) \\
\mathbf{V}_{0}(u) \\
3 \mathbf{M}(u)
\end{array}\right)
$$

By choosing the parameterization $u=\lambda(u)^{-1}$, we get the following unobservable manifold

$$
\mathbf{X}(u)=\left(\begin{array}{c}
u \mathbf{r}_{0} \\
u \mathbf{v}_{0} \\
u^{3} \mathbf{m}
\end{array}\right) .
$$

It is instructive to substitute $\mathbf{X}(u)$ into (11) and conclude that each $\mathbf{h}_{k}(\mathbf{X}(u))$ is independent of the parameter $u$, which means that all points on this manifold will give the same output $\mathbf{y}_{k}$. For example, multiplying $\mathbf{r}_{0}$ and $\mathbf{v}_{0}$ with 2 , and $\mathbf{m}$ with $2^{3}=8$ will result in the same output $\mathbf{y}_{k}$.

Furthermore, from the expression (17) we can conclude that the magnitudes of the vectors $\mathbf{r}_{0}, \mathbf{v}_{0}$ and $\mathbf{m}$ cannot be uniquely determined, only a nonlinear combination of them. However, their directions are still observable.

\subsection{Multi Sensor Observability}

The lack of observability can be solved with a second sensor. To handle multiple sensors, the sensor model has to be slightly expanded. Let the $j$ th sensor be positioned at $\boldsymbol{\theta}_{j}$. The target parameter relative to the $j$ th sensor will then be $\mathbf{x}_{j}=\left[\mathbf{r}_{0}-\boldsymbol{\theta}_{j}, \mathbf{v}_{0}, \mathbf{m}\right]^{T}$ and the total sensor model is given by

$$
\mathbf{y}_{k, j}=\mathbf{h}_{k}\left(\mathbf{x}_{j}\right)+\mathbf{e}_{k, j} \quad \text { for all } j \in J .
$$

Furthermore, in a multi-sensor scenario, the information matrices for all sensors are additive, so

$$
\mathcal{I}_{\text {tot }}\left(\mathbf{x}^{0}, \boldsymbol{\theta}_{1: J}\right)=\sum_{j=1}^{J} \mathcal{I}\left(\mathbf{x}_{j}^{0}\right) .
$$

Now, to the unobservable sensor setup presented in Table 4 a second sensor can be added. By computing the total information matrix it can be concluded that its eigenvalues are all non-zero (see Figure 4) and with that, the system is observable.

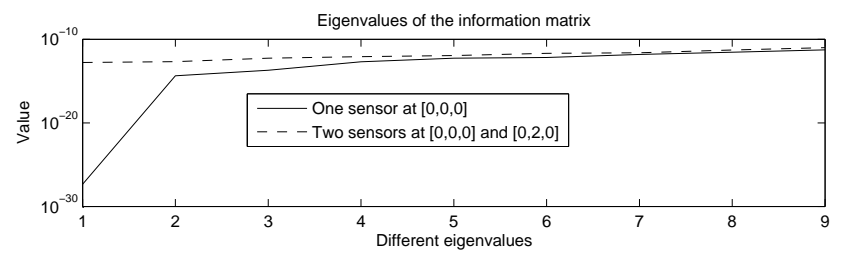

Figure 4: The eigenvalues of the information matrix for the setup presented in Table 4 being observed with 1 ) one sensor at $[0,0,0]^{T}$ and 2) two sensors at $[0,0,0]^{T}$ and $[0,2,0]^{T}$

The concept of observability can be extended by not only distinguishing between zero and non-zero eigenvalues, but also comparing their magnitudes. The design goal in the sensor deployment is then to get all eigenvalues of the same order of magnitude to achieve 
best possible observability. This property is measured by the condition number of the information matrix $\kappa\left(\mathcal{I}_{\text {tot }}\left(\mathbf{x}^{0}, \boldsymbol{\theta}_{1: J}\right)\right)$, which for a positive symmetric matrix can be expressed as

$$
\kappa(A)=\frac{\lambda_{\max }(A)}{\lambda_{\min }(A)}
$$

where $\lambda_{\max }(A)$ and $\lambda_{\min }(A)$ are maximal and minimal eigenvalues of $A$ respectively. A low condition number indicates that the system has good observability properties and its optimal value is 1 .

In order to examine where a second magnetometer should be placed to get the most well-conditioned information matrix, the condition number can be computed for different positions $\boldsymbol{\theta}_{2}$ of the second sensor. A plot illustrating this is presented in Figure 5. It can

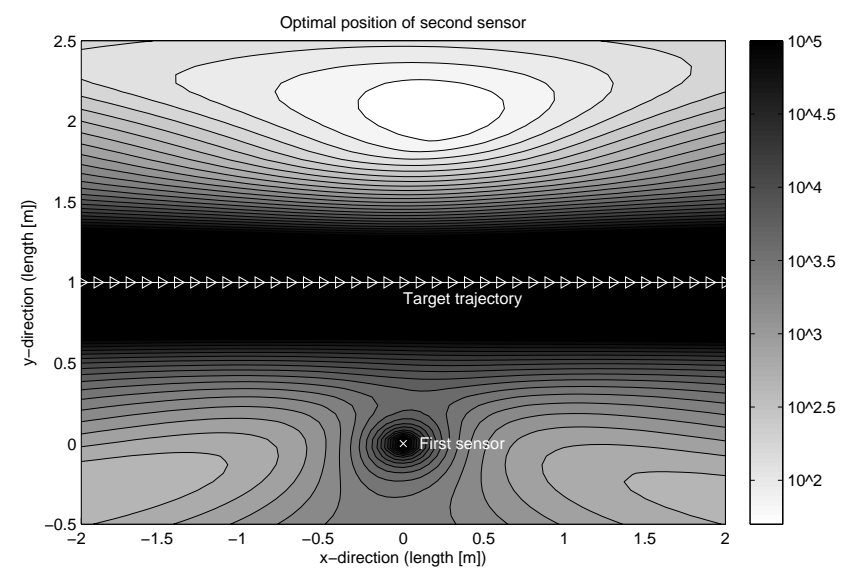

Figure 5: The condition number of the information matrix $\kappa\left(\mathcal{I}_{\text {tot }}\left(\mathbf{x}^{0}, \boldsymbol{\theta}_{1: 2}\right)\right)$ as a function of the location of the second sensor $\boldsymbol{\theta}_{2}$ in the $x$ - $y$-plane where $\mathbf{x}^{0}$ is given by the setup presented in Table 4 and $\boldsymbol{\theta}_{1}=[0,0,0]^{T}$.

be concluded that the optimal sensor deployment of a two sensor system is to locate them symmetrically at both sides of the target trajectory. If the sensors due to practical reasons must be located at the same side of the trajectory, they should be well separated, which is intuitive.

\subsection{Cramér Rao Lower Bound}

The Cramér Rao lower bound (CRLB) is related to observability, in that it applies a local analysis and basically approximates $V(\hat{\mathbf{x}})$ with a quadratic function $V(\hat{\mathbf{x}}) \approx \hat{\mathbf{x}}^{T} \mathcal{I}\left(\mathbf{x}^{0}\right) \hat{\mathbf{x}}$, where $\mathcal{I}\left(\mathbf{x}^{0}\right)$ is Fisher's information matrix (FIM), which for this problem is given by (14). The CRLB states that any unbiased estimate must have a covariance matrix larger than or equal to the inverse of the FIM

$$
\operatorname{Cov}(\hat{\mathbf{x}}) \geq \mathcal{I}\left(\mathbf{x}^{0}\right)^{-1}
$$

The design goal of the sensor deployment in the context of CRLB is then to maximize $\mathcal{I}\left(\mathbf{x}^{0}\right)$ since that will minimize the covariance matrix $\operatorname{Cov}(\hat{\mathbf{x}})$. This is achieved by minimizing

$$
\|\operatorname{Cov}(\hat{\mathbf{x}})\|_{2} \approx\left\|\mathcal{I}\left(\mathbf{x}^{0}\right)^{-1}\right\|_{2}=\frac{1}{\lambda_{\min }\left(\mathcal{I}\left(\mathbf{x}^{0}\right)\right)} .
$$

However, since the goal of this sensor deployment analysis is to find the optimal sensor positions relative to each other and the target trajectory, and not to get optimal absolute positions of the sensors (this would be achieved by localizing the sensors as close as possible to the target!), the parameters must be meaningfully normalized. By defining the characteristic length of the problem $r$ as being the the shortest euclidean distance from the target trajectory to the nearest sensor, we can introduce the following dimensionless parameters

$$
\tilde{\mathbf{r}}_{0}=\frac{\mathbf{r}_{0}}{r}, \quad \tilde{\mathbf{v}}_{0}=\frac{\mathbf{v}_{0}}{r / T}, \quad \tilde{\mathbf{m}}=\frac{\mathbf{m}}{1} .
$$

Consequently, $\mathbf{y}$ and $\mathcal{I}\left(\mathbf{x}^{0}\right)$ will be scaled accordingly

$$
\tilde{\mathbf{y}_{k}}=r^{3} \mathbf{y}_{k}, \quad \mathcal{I}\left(\tilde{\mathbf{x}}^{0}\right)=r^{6} \mathcal{I}\left(\mathbf{x}^{0}\right) .
$$

From this we get

$$
\begin{aligned}
\|\operatorname{Cov}(\hat{\tilde{\mathbf{x}}})\|_{2} & \approx \frac{1}{\lambda_{\min }\left(r^{6} \mathcal{I}\left(\mathbf{x}^{0}\right)\right)}=\frac{r^{-6}}{\lambda_{\min }\left(\mathcal{I}\left(\mathbf{x}^{0}\right)\right)} \\
& \sim \frac{\lambda_{\max }\left(\mathcal{I}\left(\mathbf{x}^{0}\right)\right)}{\lambda_{\min }\left(\mathcal{I}\left(\mathbf{x}^{0}\right)\right)}=\kappa\left(\mathcal{I}\left(\mathbf{x}^{0}\right)\right)
\end{aligned}
$$

since $\mathcal{I}\left(\mathbf{x}^{0}\right)$ decays as the signal to noise ratio, which decays as $\mathcal{O}\left(r^{-6}\right)$.

Thus, a small condition number of the information matrix will ensure a small covariance (after normalization). Therefore, the assumption in Section 4.2 that the optimal sensor deployment is measured by the condition number of the information matrix is also valid in the context of CRLB.

\section{Target Tracking Experiment}

In order to track vehicles, one does not want to exclude the possibility to incorporate process noise in the model. Furthermore, in tracking applications the best estimation at each time instance should be provided. Therefore the model (10) has to be considered and the state $\mathbf{r}_{k}, \mathbf{v}_{k}$ and $\mathbf{m}_{k}$ can at each time instant $t=k T_{s}$ be estimated using an Extended Kalman Filter.

To find the initial conditions for the EKF seems to be quite difficult if one does not know anything about the vehicle heading direction. However, with the prior knowledge that the vehicle follows a one-dimensional road, a better prediction of the initial states can be made. In order to track Vehicle 6, two EKFs have been initialized, assuming the vehicle is coming from the left and from the right respectively. With the filter 


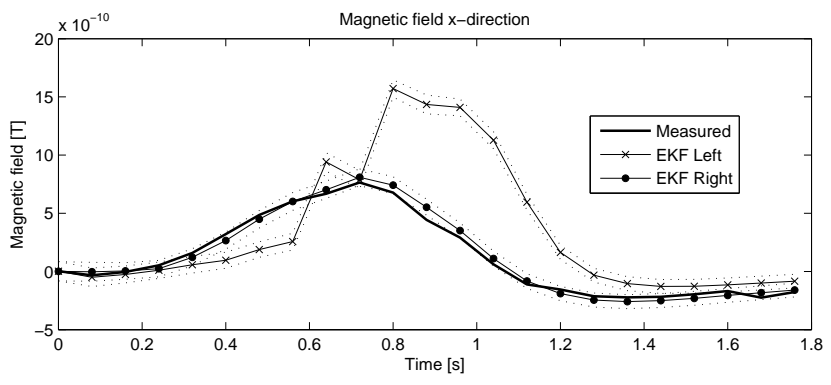

(a) The measured and predicted magnetic field in the $\mathrm{x}$-direction.

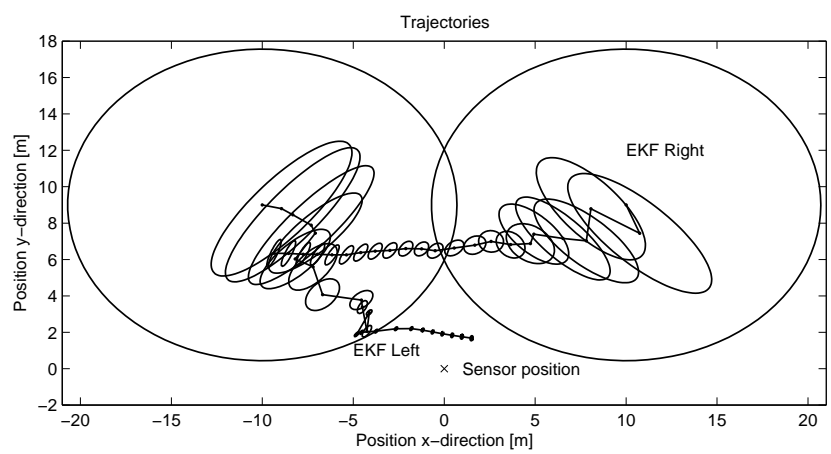

(b) The trajectories according the two different EKFs.

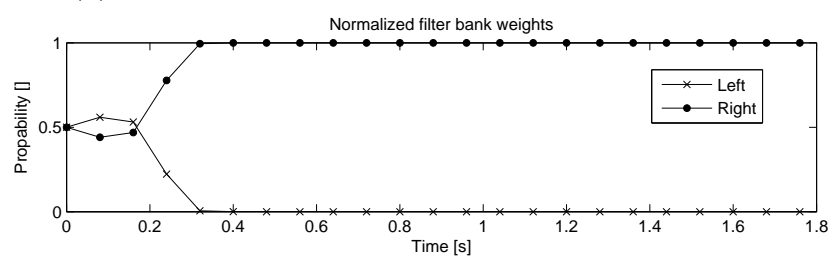

(c) The probabilities $p\left(\delta=\operatorname{Left} \mid \mathbf{y}_{1: k}\right)$ and $p\left(\delta=\operatorname{Right} \mid \mathbf{y}_{1: k}\right)$ at time instance $t=k T_{s}$.

Figure 6: Results from a tracking experiment with two differently initialized EKFs predicting the state of Vehicle 6 presented with a confidence interval of $90 \%$.

bank methodology [11] the modes $\delta=\{$ Left, Right $\}$ can be defined representing these two differently initialized EKFs. Their probabilities can now be calculated as a function of time. All results are found in Figure 6 .

Due to the nonlinearity of the model one of these filters will not converge to the correct solution. For the false one it violates the assumption that the vehicle moves in $x$-direction (see Figure $6 \mathrm{~b}$ ). Furthermore, only one filter produces a good fit with the measured data (see Figure 6a). Finally, the filter bank weights in Figure $6 \mathrm{c}$ provide the absolute measure which enables us to decide which hypothesis is correct. With these comments it can be clearly stated that this specific vehicle is coming from the right, which is correct according to Figure 3f.

\section{Conclusions and Future Work}

We have shown (theoretically and experimentally) that a moving metallic object can be modeled as a magnetic dipole if the distance to the object is large in comparison to its characteristic length. With a single magnetometer, the position, the velocity and the magnetic dipole moment of the vehicle can be estimated with high accuracy. Furthermore, it has been shown that for large objects close to the sensor this far-field model is not satisfactory. In the future, higher order terms of the model approximation will be used to design a more accurate sensor model enabling bias and variance compensation. Also, methods to classify objects will be analyzed.

\section{Acknowledgement}

The authors want to thank first Nils Jönsson for initiating the project of using magnetometers for tracking, and second Prof. Peter Münger for his expert's advices on electromagnetism.

\section{Appendix}

A magnetized vehicle can be regarded as a region with localized current density. That means, charged particles can move within the region, but neither leave it nor be added to it. This current density gives rise to an induced magnetic field outside the region (see Figure 7). With the assumption that the velocity of the vehicle is constant, this current density will be timeindependent (relative to the vehicle). This induced

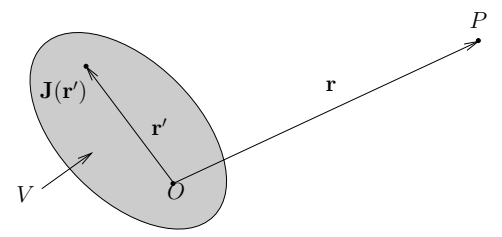

Figure 7: Localized current density $\mathbf{J}\left(\mathbf{r}^{\prime}\right)$ gives rises to a magnetic induction at the point $P$ with coordinate $\mathbf{r}$.

magnetic field, together with the homogeneous magnetic field of the earth, is measured by the magnetometer when a vehicle passes by. In order to derive an expression of this magnetic field as a function of the vector $\mathbf{r}$ from the vehicle to the observer, the electromagnetic theory has to be addressed.

All electromagnetic phenomena are governed by Maxwell's equations which are

$$
\begin{aligned}
\nabla \cdot \mathbf{E} & =\frac{\rho}{\varepsilon_{0}} \\
\nabla \times \mathbf{B}-\mu_{0} \varepsilon_{0} \frac{\partial \mathbf{E}}{\partial t} & =\mu_{0} \mathbf{J} \\
\nabla \times \mathbf{E}+\frac{\partial \mathbf{B}}{\partial t} & =0 \\
\nabla \cdot \mathbf{B} & =0,
\end{aligned}
$$

where $\mathbf{E}$ is the electric field, $\rho$ is the charge density, $\mathbf{B}$ is the magnetic field, $\mathbf{J}$ is the current density and $\varepsilon_{0} / \mu_{0}$ are the permittivity/permeability of free space. Since all currents are constant, only the static version 
of these equations has to be considered. Furthermore, only the equations concerning the magnetic field and the current density (28) and (30) are of relevance.

$$
\begin{aligned}
\boldsymbol{\nabla} \times \mathbf{B} & =\mu_{0} \mathbf{J} \\
\boldsymbol{\nabla} \cdot \mathbf{B} & =0
\end{aligned}
$$

Since $\boldsymbol{\nabla} \cdot \mathbf{B}=0$ everywhere, $\mathbf{B}$ must be the curl of some vector field $\mathbf{A}(\mathbf{r})$, called the vector potential

$$
\mathbf{B}(\mathbf{r})=\nabla \times \mathbf{A}(\mathbf{r}) .
$$

Substituting (33) into (31) gives

$$
\begin{aligned}
\boldsymbol{\nabla} \times(\boldsymbol{\nabla} \times \mathbf{A}(\mathbf{r})) & =\mu_{0} \mathbf{J} \quad \Rightarrow \\
\boldsymbol{\nabla}(\boldsymbol{\nabla} \cdot \mathbf{A})-\nabla^{2} \mathbf{A} & =\mu_{0} \mathbf{J} .
\end{aligned}
$$

Since (33) only specifies the curl of $\mathbf{A}$, the freedom of the so called gauge transformation allows one to make $\boldsymbol{\nabla} \cdot \mathbf{A}$ have any convenient functional. With the choice $\boldsymbol{\nabla} \cdot \mathbf{A}=0$, each rectangular component of the vector potential satisfies the Poisson's equation

$$
\nabla^{2} \mathbf{A}=-\mu_{0} \mathbf{J}
$$

which has the solution

$$
\mathbf{A}(\mathbf{r})=\frac{\mu_{0}}{4 \pi} \int \frac{\mathbf{J}\left(\mathbf{r}^{\prime}\right)}{\left|\mathbf{r}-\mathbf{r}^{\prime}\right|} d^{3} \mathbf{r}^{\prime}
$$

The vector potential of the induced magnetic field in Figure 7 can now be calculated by using (37) and then integrate over region of the localized current. Furthermore, the denominator in (37) can be expanded in powers of $\mathbf{r}^{\prime}$. With $r>r^{\prime}$ this will be

$$
\begin{aligned}
\frac{1}{\left|\mathbf{r}-\mathbf{r}^{\prime}\right|}= & \frac{1}{r}+\left(-\mathbf{r}^{\prime}\right)^{T} \boldsymbol{\nabla}\left(\frac{1}{r}\right)+ \\
& \frac{1}{2 !}\left(-\mathbf{r}^{\prime}\right)^{T}\left(\nabla \nabla\left(\frac{1}{r}\right)\right)\left(-\mathbf{r}^{\prime}\right)+\ldots
\end{aligned}
$$

where $r=|\mathbf{r}|$. By using this Taylor expansion in (37) one gets

$A_{i}(\mathbf{r})=\frac{\mu_{0}}{4 \pi}\left(\frac{1}{r} \int_{V} J_{i}\left(\mathbf{r}^{\prime}\right) d^{3} \mathbf{r}^{\prime}+\frac{\mathbf{r}}{r^{3}} \cdot \int_{V} J_{i}\left(\mathbf{r}^{\prime}\right) \mathbf{r}^{\prime} d^{3} \mathbf{r}^{\prime}+\ldots\right)$.

Due to the fact that the current density $\mathbf{J}(\mathbf{r})$ is localized and obeys the static continuity condition $\boldsymbol{\nabla} \cdot \mathbf{J}=0$, Gauss' theorem makes the first term in (39) zero. Furthermore, it can be shown that

$$
\mathbf{r} \cdot \int_{V} \mathbf{r}^{\prime} J_{i} d^{3} \mathbf{r}^{\prime}=(\mathbf{m} \times \mathbf{r})_{i},
$$

where $\mathbf{m}$ is the magnetic dipole moment

$$
\mathbf{m}=\frac{1}{2} \int_{V} \mathbf{r}^{\prime} \times \mathbf{J}\left(\mathbf{r}^{\prime}\right) d^{3} \mathbf{r}^{\prime}
$$

The details of these steps are clearly outlined in [1]. Using (41) in (39) gives

$$
\mathbf{A}(\mathbf{r})=\frac{\mu_{0}}{4 \pi} \frac{\mathbf{m} \times \mathbf{r}}{r^{3}} .
$$

The induced magnetic field can now be calculated directly by evaluating the curl of (42).

$$
\mathbf{B}(\mathbf{r})=\frac{\mu_{0}}{4 \pi} \frac{3(\mathbf{r} \cdot \mathbf{m}) \mathbf{r}-r^{2} \mathbf{m}}{r^{5}}
$$

When $r \gg r^{\prime}$ the truncation of the Taylor expansion in (38) makes a good approximation. Thus (43) is a good far-field model for the magnetic field induced by the vehicle.

\section{References}

[1] J. D. Jackson, Classical Electrodynamics, 2nd ed. John Wiley and Sons, Inc., 1975.

[2] R. E. Raab and O. L. de Lange, Multipole Theory in Electromagnetism. Oxford University Press Inc., New York, 2005.

[3] M. Birsan, "Non-linear kalman filters for tracking a magnetic dipole," in Proc. of Intl. Conf. on Maritime Electromagnetics, MARELEC, 2003.

[4] —, "Unscented particle filter for tracking a magnetic dipole target," in Proc. of MTS/IEE $O C E A N S, 2005$.

[5] Z. A. Daya, D. L. Hutt, and T. C. Richards, "Maritime electromagnetism and DRDC signature management research," Defence R\&D Canada, Tech. Rep., 2005.

[6] M. Birsan, "Electromagnetic source localization in shallow waters using bayesian matched-field inversion," Inverse Problems, vol. 22, no. 1, pp. 43-53, 2006.

[7] J. Callmer, M. Skoglund, and F. Gustafsson, "Electromagnetic source localization in shallow waters using bayesian matched-field inversion," Eurasip Journal on Advances in Signal Processing, 2009 .

[8] H. Gonzlez, S. R. Jurez, P. Kielanowski, and M. Loewe, "Multipole expansion in magnetostatics," Am. J. Phys., vol. 66, 1998.

[9] MTi and MTx User Manual and Technical Documentation, Xsens Technologies B.V., http://www.xsens.com, 2005.

[10] D. Törnqvist, "Statistical fault detection with applications to IMU disturbances," 2006.

[11] F. Gustafsson, Statistical Sensor Fusion, 1st ed. Studentlitteratur, 2010. 